\title{
Training the next generation of surgeons in robotic surgery
}

This article was published in the following Dove Press journal:

Robotic Surgery: Research and Reviews

2I April 2017

Number of times this article has been viewed

\section{Benjamin T Carpenter Chandru P Sundaram \\ Department of Urology, Indiana University School of Medicine, Indianapolis, IN, USA}

Correspondence: Chandru P Sundaram Department of Urology, Indiana University School of Medicine, 535 N Barnhill Dr, Suite 420, Indianapolis, IN 46032, USA

$\mathrm{Tel}+\mathrm{I} 3179483098$

Fax + I 3179440174

Email sundaram@iupui.edu
Context: Robotic surgery has been used with rapidly increasing frequency within urology and across many other surgical specialties. A standardized curriculum for the training and credentialing of robotic surgeons has unfortunately trailed far behind the adoption of this surgical technology.

Objective: To review the current available surgical skills training models, assessments, and curricula for the purpose of training resident, fellow, and practicing surgeons in an effort to promote surgical skill proficiency and mastery and to minimize the risk of patient harm.

Evidence acquisition: We performed a thorough review of available literature through a PubMed database search in February 2015.

Evidence synthesis: In this article, we compiled and scrutinized the available relevant literature regarding past and present robotic surgical training techniques and credentialing criteria. This review details the basic surgical skills (both technical and nontechnical) that are necessary for individuals and teams to be successful in the operative setting. We go on to discuss the role of current robotic surgical training techniques including dry lab and virtual simulators. Finally, we offer current validated training curricula, the Global Evaluative Assessment of Robotic Skills and Fundamentals of Robotic Surgery models, which have laid the groundwork for a future standardized model that could be applied on a national and international level and across several surgical subspecialties. The ultimate goal of the review is to provide a foundation from which a future standardized training and credentialing curriculum could be based.

Conclusion: There is currently a great need for a standardized curriculum to be developed and employed for the use of training and credentialing future and current robotic surgeons.

Keywords: robotic surgery, robotic training curriculum, robotic simulation

\section{Introduction}

The history of medicine closely follows a punctuated equilibrium model with innovation serving as the catalyst for rapid change and growth. Innovation comes in many forms, new knowledge, pharmaceutical breakthrough, novel techniques, and advanced technology.

The increasing usage of robotic surgery has been espoused ad nauseam in recent literature. It is not surprising, but no less impressive, that 1.5 million robotic surgeries have been performed throughout the world over the past decade or that $83 \%$ of prostatectomies were performed robotically in 2011 compared with just $17 \%$ only 6 years earlier (Intuitive Surgical, 2015). In the few years following 2007, the number of robotic-assisted procedures nearly tripled worldwide from 80,000 to over 200,000 (Figure 1). ${ }^{1}$ The number of da Vinci robotic surgical consoles grew 75\% between 2007 and 2009 (from 800 to 1,400 in the US and from 200 to 400 abroad). ${ }^{1}$ 


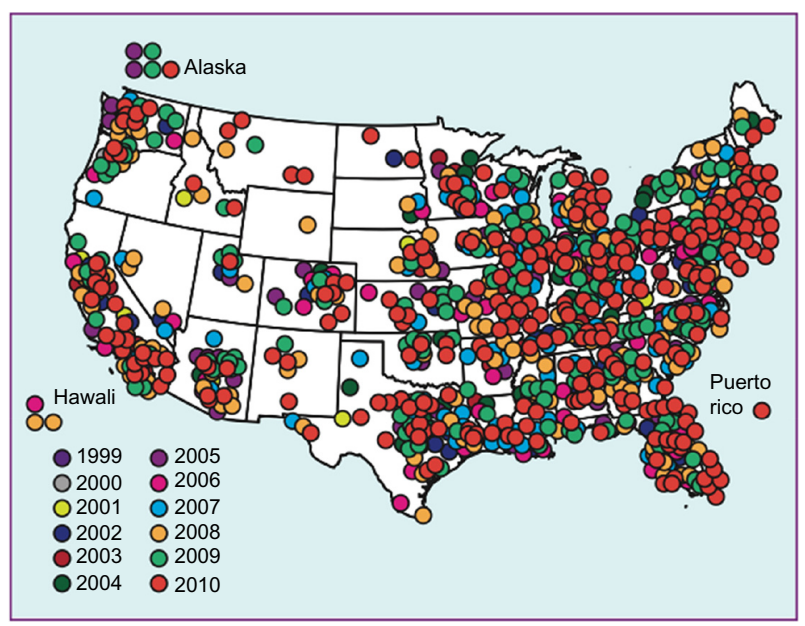

Figure I da Vinci surgical systems installed in the US, 1999-2010.' Note: Reproduced from Ahmed K, Abboudi H, Guru KA, Khan MS, Dasgupta P. Robotic surgical technology is here to stay and evolve. Trends Urol Men's Health 2013;4(2):32-36.

Robotic surgery has been widely adopted across several specialties and within urology is utilized for reconstructive, pediatric, urogynecology, and obviously oncologic procedures. In 2014 , total US procedure volume was $\sim 449,000$, of which $20 \%$ was in urology, $52 \%$ was in gynecology, and $24 \%$ was in general surgery. International procedure volume was $\sim 121,000$ in 2014, of which most procedures were in urology.

With the adoption of new technology and techniques come growing pains. It is clear that implementation of any new technique or adoption of new technology is associated with a learning curve to ensure not only proficient surgical skill, but most importantly patient safety.

This article aims to review the current available surgical skills training models, assessments, and curricula for the purpose of training resident, fellow, and practicing surgeons in an effort to promote surgical skill proficiency and mastery and to minimize the risk of patient harm.

\section{Harms}

The advent of any surgical technology comes with complications, especially in the early stages of implementation.

Cooper et $\mathrm{al}^{2}$ identified a total of 245 perioperative complications related to robotic surgery over a 12-year period (2000-2012), of which 174 were nonfatal injuries with 71 resulting in patient deaths. They also identified eight cases that were either not reported or reported incorrectly (8/245, 3\%). All parties involved in this technology have a vested interest in minimizing patient complications, especially serious and fatal complications. It becomes imperative, therefore, to identify the underlying cause of these complications so they may be corrected.

For each device-related complication (with or without patient harm), the US Food and Drug Administration requires that a report be filed and these reports are then de-identified and made available through the public Manufacturer and User Facility Device Experience database. Although this system should allow for transparency for proper evaluation of this evolving technology by surgeons, the public, and governmental agencies alike, several publications demonstrate that reporting is not standardized, is offered at the will of the device manufacturers, and ultimately may be underreported.

Andonian et $\mathrm{al}^{3}$ utilized the Manufacturer and User Facility Device Experience database to identify adverse events from robotic device failures from 2000 to 2007. A total of 189 events were identified, while only nine of these were linked to patient harm. As expected, the gross number of adverse events has increased with increased utilization of robotic surgical platforms. One could reasonably conclude that as this technology continues to evolve, device failure rates should decrease and as robotic surgical staff become more experienced they will likely be better suited to troubleshoot minor device failures. The authors demonstrated a robotic to open conversion rate related to device failure of less than $0.5 \%$, and they noted that this rate was decreased over the course of their study. It is rational to attribute this to increased technical skill and surgeon comfort related to the robotic surgical platform.

The extent of underreporting is not clear, but from these reviews, does not appear to be alarming. The risk of patient harm, however, is real even if small and it should be the continued aim of surgical training to minimize this risk through the implementation of improved training programs.

\section{General recommendations}

Much confusion and debate exists over the topic of standardizing the practice of credentialing surgeons who practice robot-assisted surgery.

The American Urologic Association (AUA) published their original Standard Operating Practices for Urologic Robotic Surgery in 2009 and has once updated this as of April 2013 (AUA SOP 2013). ${ }^{4}$ It currently states that the responsibility for credentialing surgeons ultimately lies with individual institutions. In this document, they offer a framework for training and credentialing competent robotic surgeons. It is recommended that surgeons complete an Accreditation Council for Graduate Medical Education accredited (or equivalent) residency program and that they participate in a minimum of 20 robotic cases (adult or pediatric) with a minimum of ten cases in which the resident is on the console for a key portion of the case. For urologists who do not have formal robotic surgery training, the AUA recommends completing the AUA 
Core Curriculum Fundamentals of Urologic Robotic Surgery module, completion of at least one AUA Core Curriculum module for the procedure(s) that will be the urologists' primary focus (with completion of a postmodule test and a score of $80 \%$ considered to be sufficient). The AUA goes on to recommend review of online learning modules provided by Intuitive Surgical.

Finally, the AUA makes recommendations that hands-on training including the use of inanimate exercises, animal labs, and the practice of proctoring should be utilized. There are, unfortunately, no specific recommendations regarding the types of exercises or labs or any sort of standardized assessment method for evaluating these surgeons. The vagueness within these guidelines certainly allows flexibility for credentialing institutions, however, this also introduces ambiguity and falls well short of creating the framework for a standardized national credentialing tool.

These guidelines do elucidate the two major missions of robotic curriculums: first, for use in surgical training and second, for verification of surgical proficiency and safety for the purpose of certification/credentialing. Several different platforms and proposed curricula attempt to accomplish these tasks.

\section{Surgical simulation}

One of the great tools that we currently have at our disposal for robotic training is surgical simulation. Simulations allow trainees to practice basic, but transferrable surgical skills in a safe and controlled environment. ${ }^{5,6}$ Most of the basis for our discussion and evaluation of robotic surgical training is directly established upon the work of the Fundamentals of Laparoscopic Surgery simulator training.

\section{Live simulators}

There are numerous inanimate ("dry" lab) exercises that can be used to increase trainees' basic robotic skills. Most focus on robotic exercises intended to improve dexterity and control. Fried et $\mathrm{al}^{7}$ published early and promising results using their McGill Inanimate System for Training and Evaluation of Laparoscopic Skills (MISTRIL) trainer for laparoscopy training, which was subsequently verified by results published by Sroka et al. ${ }^{8}$ These studies helped to lay the foundation that inanimate simulator training was beneficial and reproducible within surgical training. Many training institutions may offer sporadic inanimate training sessions, however, these often lack external controls and proctoring feedback for resident improvement over time.

Dulan et $\mathrm{al}^{9}$ noted the paucity of standardized, validated robotic simulation curricula and attempted to pioneer a novel program. Their curriculum consisted of an online training module provided by Intuitive Surgical followed by a half-day interactive session with a proctor designed to reinforce the concepts of the online training module. Trainees then moved onto a program of nine exercises with each trainee evaluated using the validated Fundamentals of Laparoscopic Surgery approach. A trainee would then repeat the exercises over time until two consecutive proficient scores were demonstrated. This model is reproducible and its development was reported to cost just over $\$ 2,200$. It does, however, require some significant time and energy from a team for initial setup as well as requiring use of the robotic console and instruments either during nonoperative hours or through a dedicated lab console. Arain et a $1^{10}$ utilized this same model with 55 different trainees. They were able to demonstrate significant trainee performance improvement as well as feasibility and reliability.

Animal or cadaveric (so-called wet labs) can be an invaluable training tool. They allow trainees to gain valuable experience with actual human anatomy and tissue or a similar equivalent without putting any human patients in danger. The major drawback to these labs is that they are expensive and labor intensive as they rely on costly procurement and proper management of animal or cadaveric subjects as well as the use of a borrowed or dedicated lab robotic console, and finally expendables such as robotic instruments, staples, clips, and suture.

\section{Virtual simulators}

Early comparison studies of virtual reality training were based on laparoscopic techniques, but showed definite positive results. Seymour ${ }^{11}$ demonstrated residents who trained on a virtual reality platform were faster and less likely to cause injury during subsequent human laparoscopic cholecystectomy dissections when compared to trainees who had only standard (no virtual reality) training.

Two of the most widely available early commercial robotic virtual trainer platforms were the Robotic Surgery Simulator and the $\mathrm{dV}$-Trainer ${ }^{\mathrm{TM}}$. Both of these models employ stand-alone consoles made to imitate the hand and foot controls of the da Vinci system. Both require significant capital outlay of about $\$ 90-125,000$ for the Robotic Surgery Simulator system and \$85-100,000 for the dV-Trainer (both requiring annual service contracts). A third, less expensive, model is the SimSurgery education platform at about $\$ 40,000$ (with an annual service contract). Although less expensive, this platform does not offer dedicated urology modules and does not have an actual simulator console making it more similar to a desktop computer. ${ }^{1}$ 
Table I Comparison of virtual reality simulators ${ }^{12}$

\begin{tabular}{lllll}
\hline & $\begin{array}{l}\text { Face } \\
\text { validation }\end{array}$ & $\begin{array}{l}\text { Skill exercises with } \\
\text { content validation }\end{array}$ & $\begin{array}{l}\text { Performance metrics } \\
\text { with construct validation }\end{array}$ & $\begin{array}{l}\text { Procedural } \\
\text { modules }\end{array}$ \\
\hline RoSS & $\checkmark$ & $\checkmark$ & & $\checkmark$ \\
dV-Trainer & $\checkmark$ & $\checkmark$ & $\checkmark$ & $\$ 100-125,000$ \\
SEP Robot & & & $\checkmark$ & $+1-$ \\
dVSS & & $\checkmark$ & $\checkmark$ & $\$ 40-100,000$ \\
\hline
\end{tabular}

Note: + I-, capability present, but no urologic procedural components available. Reproduced with permission from Lallas CD, Davis JW, Members of The Society of Urologic Robotic Surgeons. Robotic surgery training with commercially available simulation systems in 20I I: a current review and practice pattern survey from the Society of Urologic Robotic Surgeons. J Endourol. 2012;26(3):283-293, () 2012 Mary Ann Liebert Inc,. The publisher for this copyrighted material is Mary Ann Liebert, Inc. publishers. ${ }^{2}$

Abbreviations: dVSS, da Vinci Skills Simulator; RoSS, Robotic Surgery Simulator; SEP, SimSurgery education platform.

One platform gaining wide popularity is a combined effort of Intuitive Surgical and Mimic Technologies. They collaborated to create the da Vinci Skills Simulator. This is an integration of the dV-Trainer software made available on a "backpack" on the existing console that utilizes the hardware of the existing da Vinci console. The cost is similar to that of the other more expensive virtual reality simulators.

Hung et $\mathrm{l}^{13}$ demonstrated validity for the da Vinci Skills Simulator. In this study, 24 trainees performed baseline simulator performance and then baseline performance on three exercises performed on ex vivo animal tissue. Then one group completed an eight to ten training curriculum using the Skills Simulator. At the conclusion of the study period, the two groups repeated the ex vivo tissue exercises. They found that there was significant improvement in the simulator group compared with the control group. Furthermore, these results were verified by a similar curriculum published by Crochet et al ${ }^{14}$ (Table 1). ${ }^{12,16}$

\section{Nontechnical skills (team building)}

Nontechnical skills are a major element of surgical training and one that is often neglected when formulating and reviewing components of a training curriculum. Perhaps, the most important aspect of nontechnical skills is team building/ communication.

A recent review of surgical and psychological literature identified six core skills required by all surgeons to be able to operate effectively and safely. ${ }^{16}$

- Communication

- Situational awareness

- Decision making

- Task management

- Team work

- Leadership

While the Observational Teamwork Assessment for Surgery and the Oxford Nontechnical Skills both show excellent construct validity and content, it is cautioned that these (and similar assessment tools) require further investigation to assess their implementation within training curricula. One of the major barriers to these assessments is their need for surgical faculty to assess trainees' nonsurgical skills. Recent studies ${ }^{17,18}$ have demonstrated that experienced surgeons often significantly lack insight into their own nontechnical behaviors, making them very suboptimal evaluators for trainees.

The authors of the Fundamentals of Robotic Surgery program (see Example Cirricula section) outline seven traits of team building and communication they describe as vital for safe and effective operative teams. ${ }^{11}$ These traits were then applied to three different categories: preoperative, intraoperative, and postoperative team tasks. This allows for the formation of a checklist of various communication skills required in different scenarios for successful surgical training. They acknowledge that a surgical team could utilize already existing programs such as Team Strategies and Tools to Enhance Performance and Patient Safety (TeamSTEPPS), but stress that regardless of the program, a checklist of vital team functions should be formulated and closely assessed.

\section{Example curricula}

Expertise is not simply achieved from background education and extensive experience. Instead, it is attained through the use of complex systems (curricula) that allow for execution, monitoring, and analyses of performance. ${ }^{15}$

Structuring a standardized curriculum for robotic surgery trainees must incorporate many different aspects of surgical training, all with patient safety as the ultimate goal. It seems logical and equally feasible to first expose trainees to online learning modules to establish the theoretical aspect of robotic training. Trainees would then progress onto virtual trainers to teach them fundamental mechanics and maneuvers essential to robotic surgery. Finally, trainees would advance onto live animal labs and proctored human surgery once they are deemed competent. Creating such a complex curriculum is by no means an easy task.

Shepherd et al ${ }^{19}$ set out to review the available robotic surgical training curricula and to comment on the available 
validation studies for various existing training tools. They selected 20 available studies that evaluated existing robotic surgery training curricula and validated tools. From a careful analysis of these studies the authors offer a framework for potential curricula that should utilize simulation-based training curricula as the backbone of modern surgical training programs. ${ }^{5,6}$ Simulation-based programs allow for training to conform to tighter training work hour restrictions, new technology, and increasing focus on surgical outcomes.

Goh et $\mathrm{al}^{20}$ did some foundational work with their Global Evaluative Assessment of Robotic Skills. The concept was based on the previously described Global Operative Assessment for Laparoscopic Skills tool. With this new tool, six fundamental robotic skill domains are assessed on a 5-point Likert scale. Using robotic prostatectomy as the index procedure, 25 trainees and four attending surgeons were evaluated by a panel of four observers. This study demonstrated excellent internal consistency and accuracy able to differentiate different robotic skill levels.

A recently published study offers an external validation of this tool. ${ }^{21}$ In the study by Aghazadeh et al, "expert" and "novice" participated in standardized robotic surgical tasks in an in vivo porcine model. The Global Evaluative Assessment of Robotic Skills tool was able to accurately differentiate between skill levels, demonstrating both consistency and reliability. This tool proves to be much more accurate than simply using number of cases performed to determine surgical expertise and therefore offers great utility as a possible basis for a robotic surgery curriculum.

Arguably the most comprehensive robotic surgerytraining curriculum constructed to date is the Fundamentals of Robotic Surgery program..$^{22}$ The Smith et al curriculum came about from a consortium of 14 leading international surgical societies that met over the course of a series of consensus conferences. This group managed to first identify 25 outcome measurements felt to encompass the necessary basic robotic surgery skills. They went on to formulate a teaching curriculum for the instruction of safe and efficient robotic surgeons. Their proposed curriculum is nicely broken down into three main categories: cognitive skills, psychomotor skills, and team training.

Cognitive skills, or knowledge base, is the didactic component of the curriculum that covers basic principles and functionality of the robot including setup, intraoperative functionality, and postoperative breakdown. The psychomotor component of the curriculum identified ten tasks that utilized the necessary basic technical skills and furthermore developed a single integrated device on which all of these tasks could be performed and evaluated. They dubbed this the Fundamentals of Robotic Surgery dome device (Figure 2). The third and final prong of the curriculum is team training and communications. This is detailed in the previous section.

Finally, the authors urge that regardless of the exact curriculum utilized, it should be based on achieving preset goals to prove learner competency and not based on a finite timetable. Unfortunately, many current programs are set up in this exact manner.

\section{Conclusion}

While the utilization of robotic surgical technology has experienced exponential growth in many parts of the world and across numerous specialties, standardized training and credentialing of robotic surgeons unfortunately remains in a nascent state. The need for a standardized robotic training curriculum is much needed and long overdue. The lack of a standardized training curriculum lends itself to serious disparity in the quality of robotic training depending on trainee location and specialty. We have shown that multiple validated training tools exist, as does the framework for such a curriculum.

We believe that such a curriculum should encompass knowledge base (cognitive skill), tactile skill (psychomotor), and nontechnical skills (communication/team building). The basis for this curriculum should be surgical simulation, as it allows for safe acquisition of the necessary surgical skill in a controlled environment and then transference of this skill into live surgery. A curriculum should also be based on trainees achieving and demonstrating competence through identifiable outcome measures rather

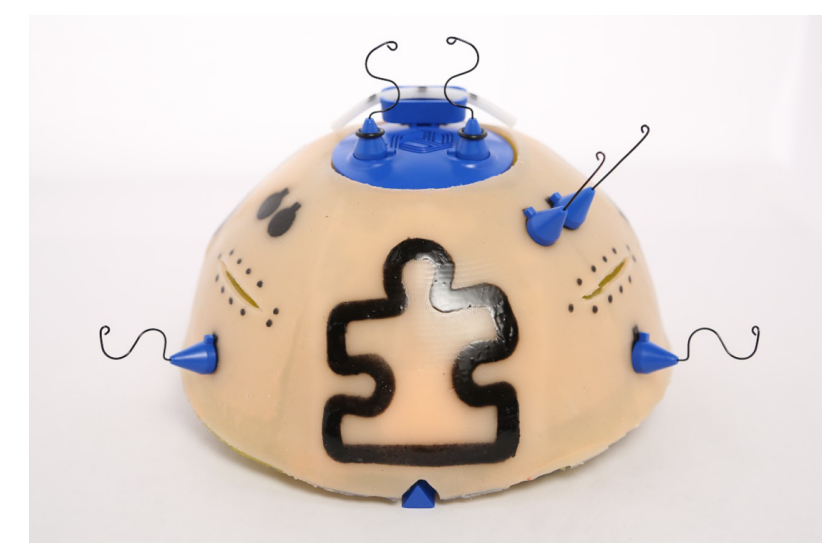

Figure 2 Fundamentals of Robotic Surgery dome device. Image courtesy of Roger Smith, PhD. Chief Technology Office at Nicholson Center in Florida Hospital, Celebration, FL, USA 
than achieving a preset duration of training exercises. This ultimately allows for a higher level of competence in skill among surgical trainees and helps to minimize surgical harm to patients.

We applaud the work of the authors highlighted above and greatly look forward to assisting in the development of this much-needed and long overdue robotic surgery training curriculum.

\section{Disclosure}

The authors report no conflicts of interest in this work.

\section{References}

1. Ahmed K, Abboudi H, Guru KA, Khan MS, Dasgupta P. Robotic surgical technology is here to stay and evolve. Trends Urol Men's Health. $2013 ; 4: 32-36$.

2. Cooper MA, Ibrahim A, Lyu H, Makary MA. Underreporting of robotic surgery complications. J Healthc Qual. 2015;37;133-138.

3. Andonian S, Okeke Z, Okeke DA. Device failures associated with patient injuries during robot-assisted laparoscopic surgeries: a comprehensive review of FDA MAUDE database. Can J Urol. 2008;15: 3912-3916.

4. American Urological Association. Standard Operating Practices (SOP'S) For Urologic Robotic Surgery. Linthicum, MD: American Urological Association; 2013. Available from: https:/www.auanet. org/common/pdf/about/SOP-Urologic-Robotic-Surgery.pdf. Accessed February 15, 2017.

5. Seymour NE, Gallagher AG, Roman SA, et al. Virtual reality training improves operating room performance: results of a randomized, doubleblinded study. Ann Surg. 2002;236(4):458-463.

6. Ericsson KA. Deliberate practice and acquisition of expert performance: a general overview. Acad Emerg Med. 2008;15(11):988-994.

7. Fried GM, Feldman LS, Vassiliou MC, et al. Proving the value of simulation in laparoscopic surgery. Ann Surg. 2004;240(3): $518-525$.

8. Sroka G, Feldman LS, Vassiliou MC, Kaneva PA, Fayez R, Fried GM. Fundamentals of laparoscopic surgery simulator training to proficiency improves laparoscopic performance in the operating room a randomized controlled trial. Am J Surg. 2010;199(1):115-120.

9. Dulan G, Rege RV, Hogg DC, Gilberg-Fisher KK, Tesfay ST, Scott DJ. Content and face validity of a comprehensive robotic skills training program for general surgery, urology, and gynecology. Am J Surg. 2012;203(4):535-539.
10. Arain NA, Dulan G, Hogg DC, et al. Comprehensive proficiency-based inanimate training for robotic surgery: reliability, feasibility, and educational benefit. Surg Endosc. 2012;26(10):2740-2745.

11. Seymour NE. VR to OR: a review of the evidence that virtual reality simulation improves operating room performance. World J Surg. 2008;32(2):182-188.

12. Lallas CD, Davis JW, Members of The Society of Urologic Robotic Surgeons. Robotic surgery training with commercially available simulation systems in 2011: a current review and practice pattern survey from the Society of Urologic Robotic Surgeons. J Endourol. 2012;26(3):283-293.

13. Hung AJ, Patil MB, Zehnder P, et al. Concurrent and predictive validation of a novel robotic surgery simulator: a prospective, randomized study. J Urol. 2012;187(2):630-637.

14. Crochet P, Aggarwal R, Dubb SS, et al. Deliberate practice on a virtual reality laparoscopic simulator enhances the quality of surgical technical skills. Ann Surg. 2011;253(6):1216-1222.

15. Bahler CD, Sundaram CP. Training in robotic surgery. Urol Clin North Am. 2014;41(4):581-589.

16. Yule S, Rowley D, Flin R, Maran N, Youngson G, Duncan J, PatersonBrown S. Experience matters: comparing novice and expert ratings of non-technical skills using the NOTSS system. ANZ J Surg. 2009;79:154-160

17. Arora S, Miskovic D, Hull L, Moorthy K, Aggarwal R, Johannsson H, Gautama S, Kneebone R, Sevdalis N. Self vs expert assessment of technical and non-technical skills in high fidelity simulation. Am J Surg. 2011;202:500-506.

18. Hull L, Arora S, Kassab E, Kneebone R, Sevdalis N. Observational teamwork assessment for surgery: content validation and tool refinement. J Am Coll Surg. 2011;212:234-243.e1-5

19. Shepherd W, Arora KS, Abboudi H, Shamim Khan M, Dasgupta P, Ahmed K. A review of the available urology skills training curricula and their validation. J Surg Educ. 2014;71(3):289-296.

20. Goh AC, Goldfarb DW, Sander JC, Miles BJ, Dunkin BJ. Global evaluative assessment of robotic skills: validation of a clinical assessment tool to measure robotic surgical skills. J Urol. 2012;187(1):247-252.

21. Aghazadeh MA, Jayaratna IS, Hung AJ, et al. External validation of Global Evaluative Assessment of Robotic Skills (GEARS). Surg Endosc. 2015;29:3261-3266.

22. Smith R, Patel V, Satava R. Fundamentals of robotic surgery: a course of basic robotic surgery skills based upon a 14-society consensus template of outcomes measures and curriculum development. Int J Med Robot. 2014;10(3):379-384.
Robotic Surgery: Research and Reviews

\section{Publish your work in this journal}

Robotic Surgery: Research and Reviews is an international, peer reviewed, open access, online journal publishing original research, commentaries, reports, and reviews on the theory, use and application of robotics in surgical interventions. Articles on the use of supervisorycontrolled robotic systems, telesurgical devices, and shared-control

\section{Dovepress}

systems are invited. The manuscript management system is completely online and includes a very quick and fair peer review system, which is all easy to use. Visit http://www.dovepress.com/testimonials.php to read real quotes from published authors. 